\title{
苯并香豆素硫腿类受体分子的设计、合成与阴离子识别研究
}

\author{
晏希泉 ${ }^{a}$ 卓继斌 ${ }^{a}$ 王吉成 ${ }^{a}$ 谢莉莉 $a, b$ 袁耀锋 $*, a, b$ \\ $\left({ }^{a}\right.$ 福州大学化学学院 福州 350116) \\ ( ${ }^{b}$ 中国科学院福建物质结构研究所 结构化学国家重点实验室 福州 350002)
}

\begin{abstract}
摘要 以 $\beta$-菜酚为原料, 经过多步反应合成了 5 种不同电子效应的 1-(5,6-苯并香豆素-3-甲酰基)-4-芳基氨基硫腿衍生 物, 并通过 ${ }^{1} \mathrm{H} \mathrm{NMR},{ }^{13} \mathrm{C} N \mathrm{NR}, \mathrm{IR}$ 及元素分析等测试手段对受体分子进行表征. 利用紫外一可见吸收光谱和苂光光谱考 察了这类受体分子与 $\mathrm{F}^{-}, \mathrm{AcO}^{-}, \mathrm{Cl}^{-}, \mathrm{Br}^{-}, \mathrm{I}^{-}, \mathrm{HSO}_{4}{ }^{-}$等阴离子的作用, 发现受体分子对其它阴离子无响应; 而对 $\mathrm{F}^{-}$和 $\mathrm{AcO}^{-}$离子具有良好的选择性检, 检测限分别为 5.45 与 $7.00 \mu \mathrm{g} / \mathrm{L}$, 响应时间分别为 5 与 $10 \mathrm{~min}$. 实验结果表明该类受体 分子与阴离子通过氢键形成配位物, 从而导致光谱信号的变化. 通过主客体间结合比和稳定常数的计算, 证实了受体 分子对 $\mathrm{F}^{-}$和 $\mathrm{AcO}^{-}$选择性好, 形成稳定化合物. Job's 曲线可知受体分子与阴离子形成 $1: 2$ 的配合物, 并利用核磁滴定 与理论计算 $[\mathrm{B} 3 \mathrm{LYP} / 6-31 \mathrm{G}(\mathrm{d}, \mathrm{p})]$ 进一步证实了受体分子与阴离子的氢键作用.
\end{abstract}

关键词 苯并香豆素; 硫艮; 阴离子识别; 氢键

\section{Design, Synthesis and Anion Recognition of Benzocoumarin-Based Thiourea Receptors}

\author{
Yan, Xiquan ${ }^{a} \quad{\text { Zhuo, } \text { Jibin }^{a} \quad \text { Wang, Jicheng }}^{a} \quad$ Xie, Lili ${ }^{a} \quad$ Yuan, Yaofeng ${ }^{*, a, b}$ \\ ( ${ }^{a}$ College of Chemistry, Fuzhou University, Fuzhou 350116) \\ $\left({ }^{b}\right.$ State Key Laboratory of Structural Chemistry, Fujian Institute of Research on the Structure of Matter, \\ Chinese Academy of Sciences, Fuzhou 350002)
}

\begin{abstract}
Anions display a great significant role in the environment and life science. The design and synthesis of receptors with high selective recognition anion has attracted considerable attention. The selective identification fluoride anion of benzocoumarin-based thiourea receptors $\mathbf{4 a} \sim \mathbf{4} \mathbf{e}$ were obtained starting from $\beta$-naphthol by a multi-step reaction. Their structures were characterized by ${ }^{1} \mathrm{H}$ NMR, ${ }^{13} \mathrm{C}$ NMR, IR and elemental analysis. The interactions between receptors $4 \mathbf{a} \sim 4 \mathbf{e}$ and anions $\left(\mathrm{F}^{-}, \mathrm{AcO}^{-}, \mathrm{Cl}^{-}, \mathrm{Br}^{-}, \mathrm{I}^{-}, \mathrm{HSO}_{4}^{-}\right.$) were studied by optical (UV-Vis absorption and fluorescence) and ${ }^{1} \mathrm{H}$ NMR spectroscopy. The results showed that the receptors with anion through hydrogen bonds formed stable $1: 2$ complexes, which leaded to the change spectroscopy of receptor molecules. Determination of the combined ratio and the stability constants of complexes were borne out receptor molecules with high alternative recognition $\mathrm{F}^{-}$or $\mathrm{AcO}^{-}$anions with limit of detect of $5.45 \mathrm{and} 7.00 \mu \mathrm{g} / \mathrm{L}$ and the effect of reaction time of $5 \mathrm{~min}$ and $10 \mathrm{~min}$ respectively. However, there is no selectively responds to other anions. Using NMR titration and theoretical (B3LYP/6-31G $(\mathrm{d}, \mathrm{p})$ ) methods further confirmed the hydrogen bond acceptor molecules and anions.
\end{abstract}

Keywords benzo coumarin; thiourea; anion recognition; hydrogen bond

阴离子在环境和生命科学中起着重要作用, 设计合 成对某特定阴离子具有选择性识别的受体分子有十分 重要意义 ${ }^{[1,2]}$. 氟离子作为人体必需元素之一, 可以作为 食品添加剂, 对骨骼保健具有相当重要的作用; 但是人
体摄入过量氟离子会引起骨质疏松症、骨肉瘤等疾 病 $^{[3,4]}$. 近年来，人们已经合成了一系列以酰胺、吲哚、 吡咯、硫嫝等为识别位点的受体分子, 这些受体分子能 够选择性地识别氟离子, 并取得了很好的效果 ${ }^{[5]}$.

\footnotetext{
*E-mail: yaofeng_yuan@fzu.edu.cn

Received May 26, 2015; revised June 12, 2015; published online June 19, 2015.

Project supported by the National Natural Science Foundation of China (No. J1103303), the State Key Laboratory of Structural Chemistry, Fujian Institute of Research on the Structure of Matter, Chinese Academy of Sciences (No. 20120010).

国家基础科学人才培养基金(No. J1103303)、中国科学院福建物质结构研究所结构化学国家重点实验室(No. 20120010)资助项目.
} 
香豆素类衍生物是自然界中广泛存在的一类杂环 化合物. 它的母体结构为肉桂酸内酯, 具有良好的光学 特性, 是优秀荧光候选团. 它常被用于苂光指示剂、苂

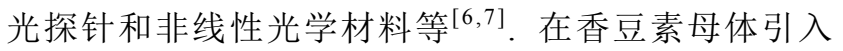
“推-拉” 电子效应的取代基，可有望改善该类化合物的 苂光性质. 近年来, 国内对香豆素类荧光探针的研究十 分重视, 逐渐成为一个阴离子识别的新领域 $[9,10]$. 硫嫝 类衍生物含有两个 $\mathrm{N}-\mathrm{H}$ 基团, 具有强的氢键质子供体 特征和路易斯酸的行为特点, 作为阴离子受体时能够与 氟离子通过双重氢键形成稳定的配合物. 因此, 硫脲类 受体分子对氟离子的识别研究成为当前研究的一大热 点 ${ }^{[1]}$. 本文以苯并香豆素作为信号基团, 以硫䏱作为桥 联基团设计合成可裸眼识别氟离子的探针分子, 利用紫 外吸收光谱和荧光吸收光谱研究传感分子与氟离子相 互作用时光谱变化, 并利用核磁技术探究其作用机理, 为发展高效灵敏的氟离子检测技术提供理论依据.

\section{1 结果和讨论}

\section{1 合成目标化合物研究}

目标受体分子 $4 \mathrm{a} \sim 4 \mathrm{e}$ 的合成路线如 Scheme 1 所示. 在制备中间产物 2 时, 由于其不稳定, 有刺激性气味, 纯化与表征困难. 因此采用将未经处理的 $\mathbf{2}$ 与水合肼直 接反应得到高产率的中间产物 $\mathbf{3}$, 通过核磁氢谱表征, 证明了产物为酰肼类化合物 3. 本文合成另一大难点是 合成的目标受体分子 $4 \mathrm{a} \sim 4 \mathrm{e}$ 的溶解度小并且极性大, 微溶于二氯甲烷, 可溶于 DMSO 中, 对目标分子的分离 纯化难度大.
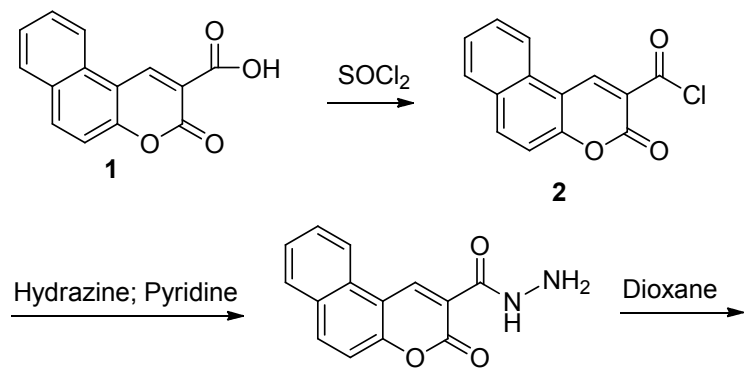

3

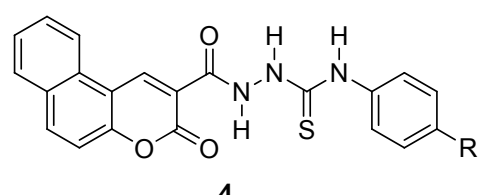

4a $\mathrm{R}=\mathrm{H}$

4b $\mathrm{R}=\mathrm{CH}_{3}$

$4 c \mathrm{R}=\mathrm{CN}$

4d $\mathrm{R}=\mathrm{OCH}_{3}$

$4 \mathrm{e}=\mathrm{NO}_{2}$

图式 1 目标分子 $4 \mathrm{a} \sim 4 \mathrm{e}$ 的合成路线

Scheme 1 Synthetic route of receptors $4 a \sim 4 e$

\section{2 光谱性能研究}

\subsection{1 紫外滴定研究}

利用紫外可见分光光度仪研究受体分子 $4 \mathrm{a} \sim 4 \mathrm{e}$ 与 各阴离子 $\left(\mathrm{F}^{-}, \mathrm{Cl}^{-}, \mathrm{Br}^{-}, \mathrm{I}^{-}, \mathrm{HSO}_{4}^{-}\right.$和 $\left.\mathrm{AcO}^{-}\right)$的紫外吸收 变化. 在不加阴离子时, 受体分子 $\mathbf{4 a} \sim \mathbf{4 e}$ 均在约 385 $\mathrm{nm}$ 处有吸收峰, 加入 $\mathrm{F}^{-}$或 $\mathrm{AcO}^{-}$时均能引起受体分子 $4 \mathrm{a} \sim 4 \mathrm{e}$ 吸收光谱的显著变化, 该过程属于 ICT 过程. 但 是对于 $\mathrm{Cl}^{-}, \mathrm{Br}^{-}, \mathrm{I}^{-}$及 $\mathrm{HSO}_{4}^{-}$, 紫外吸收光谱几乎没响 应，说明受体分子对 $\mathrm{F}^{-}$和 $\mathrm{AcO}^{-}$有紫外信号响应显著. 以受体分子 $4 \mathbf{a}$ 为例，图 1 为该受体分子加入不同浓度的 $\mathrm{F}^{-}$或 $\mathrm{AcO}^{-}$阴离子后吸收光谱的变化. 随着加入 $\mathrm{F}^{-}$或 $\mathrm{AcO}^{-}$后, 在 $385 \mathrm{~nm}$ 处, 吸光度逐渐减小, 在 $400 \sim 500$ $\mathrm{nm}$ 区域出现三个新的吸收峰，此峰为新形成配合物的 吸收峰. 而加入 $\mathrm{Cl}^{-}, \mathrm{Br}^{-}, \mathrm{I}^{-}$和 $\mathrm{HSO}_{4}{ }^{-}$四种阴离子时, 在 $385 \mathrm{~nm}$ 处只有略微下降，在 $400 \sim 500 \mathrm{~nm}$ 区域也只有略 微上升，没有新的峰的出现. 同时可以明显观察到在 $400 \mathrm{~nm}$ 处有一个清晰的等吸收点, 说明有稳定配合物 生成.
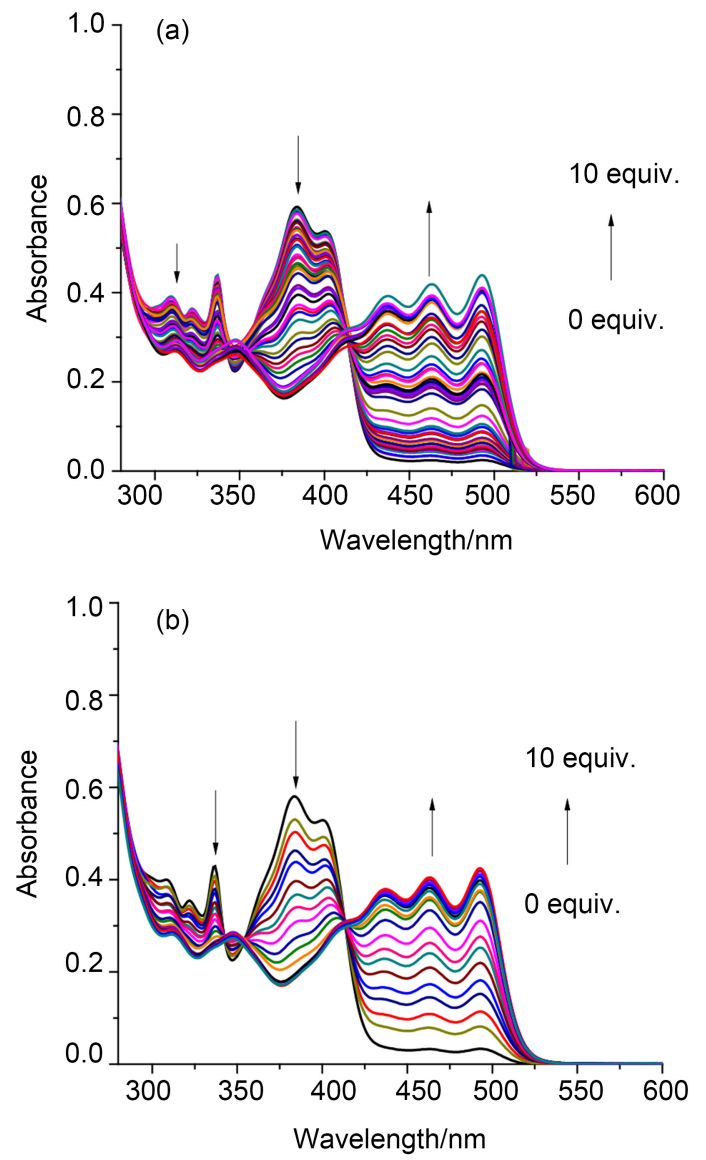

图 1 受体分子 $4 \mathrm{a}\left(c=2.5 \times 10^{-5} \mathrm{~mol} \cdot \mathrm{L}^{-1}\right)$ 在 DMSO 溶液的紫 外滴定曲线图

Figure 1 UV-Vis spectral titration of receptor $4 \mathbf{a}\left(c=2.5 \times 10^{-5}\right.$ $\mathrm{mol} \cdot \mathrm{L}^{-1}$ ) in DMSO solution

(a) $\mathrm{F}^{-}$; (b) $\mathrm{AcO}$ 


\subsection{2 “裸眼识别”}

将受体分子 $4 \mathbf{a}$ 配制成 $2.5 \times 10^{-5} \mathrm{~mol} \cdot \mathrm{L}^{-1}$ 的 DMSO 溶液中, 分别滴加 5 equiv. 的 $\mathrm{F}^{-}, \mathrm{Cl}^{-}, \mathrm{Br}^{-}, \mathrm{I}^{-}, \mathrm{HSO}_{4}{ }^{-}$和 $\mathrm{AcO}^{-}$六种离子的四丁基铵溶液. 由图 2 可得, 加入 $\mathrm{F}^{-}$或 $\mathrm{AcO}^{-}$后, 溶液由无色变为黄色, 而加入其它阴离 子, 溶液的颜色没有发生变化. “裸眼实验” 结果初步 说明了受体分子 $\mathbf{4 a}$ 可以定性地将 $\mathrm{F}^{-}$和 $\mathrm{AcO}^{-}$与其它阴离 子 $\left(\mathrm{HSO}_{4}{ }^{-}, \mathrm{Cl}^{-}, \mathrm{Br}^{-}\right.$和 $\left.\mathrm{I}^{-}\right)$区分开来. 向受体分子 $4 \mathrm{e}$ 逐步 滴加至 100 equiv. 的 $\mathrm{F}^{-}$, 溶液颜色依次由浅褐色变化到 血红色, 如图 3. 这是由于受体分子 $4 \mathrm{e}$ 带有强吸电子基 才, 加入 $\mathrm{F}^{-}$使受体分子去质子, 促进分子内电荷迁移 (ICT), 因而溶液颜色变化到血红色.

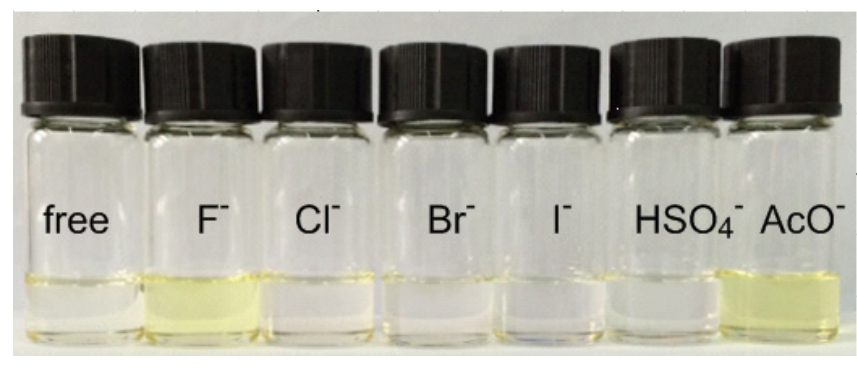

图 2 受体分子 $4 \mathrm{a}\left(c=2.5 \times 10^{-5} \mathrm{~mol} \cdot \mathrm{L}^{-1}\right)$ 在 $\mathrm{DMSO}$ 溶液中, 加入六种阴离子后溶液颜色的变化.

Figure 2 Color changes of receptor $4 \mathbf{a}\left(c=2.5 \times 10^{-5} \mathrm{~mol} \cdot \mathrm{L}^{-1}\right)$ in DMSO solution after adding to six anions

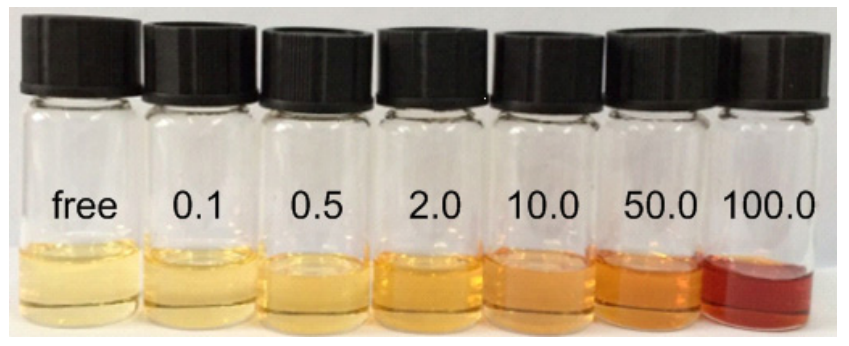

图 3 受体分子 $4 \mathrm{e}\left(c=2.5 \times 10^{-5} \mathrm{~mol} \cdot \mathrm{L}^{-1}\right)$ 在 DMSO 溶液中加 入不同倍摩尔量的 $\mathrm{F}^{-}$阴离子后溶液颜色的变化

Figure 3 Color changes of receptor $4 \mathbf{e}\left(c=2.5 \times 10^{-5} \mathrm{~mol} \cdot \mathrm{L}^{-1}\right)$ in DMSO solution after addition $\mathrm{F}^{-}$ion

From left to right adding $\mathrm{F}^{-}$ion: $0,0.1,0.5,2.0,10.0,50.0,100.0$ equiv.

\subsection{3 荧光光谱的测定}

通过采用苂光滴定法研究了不同受体分子 $4 \mathrm{a} \sim 4 \mathrm{e}$ 及其与不同阴离子 $\left(\mathrm{F}^{-}, \mathrm{AcO}^{-}, \mathrm{Cl}^{-}, \mathrm{Br}^{-}, \mathrm{I}^{-}, \mathrm{HSO}_{4}{ }^{-}\right)$之间 的相互作用. 将受体分子配制成浓度为 $2.5 \times 10^{-5} \mathrm{~mol}$ $\mathrm{L}^{-1}$ 的 DMSO 溶液, 阴离子 $\left(\mathrm{F}^{-}, \mathrm{AcO}^{-}, \mathrm{Cl}^{-}, \mathrm{Br}^{-}, \mathrm{I}^{-}\right.$, $\mathrm{HSO}_{4}{ }^{-}$) 四丁基铵盐均配制成 $2.5 \times 10^{-3} \mathrm{~mol} \cdot \mathrm{L}^{-1}$. 受体 分子的浓度保持不变, 在 $410 \mathrm{~nm}$ 的激发波长下, 向受体 分子中滴加不同阴离子时, 测定其苂光光谱变化. 以 $\mathbf{4 a}$ 为例, 受体分子与阴离子作用前后荧光光谱测定的结果
表明: 加入 $\mathrm{F}^{-}$或 $\mathrm{AcO}^{-}$后，主体的苂光强度明显增强. 这是因为受体分子与 $\mathrm{F}^{-}$形成配合物, 使得受体分子去 质子化, 电荷密度上高, 从而导致苂光增强 ${ }^{[12]}$; 加入 $\mathrm{AcO}^{-}$时, 受体分子发生扭转进行配位, 刚性增强, 导致 受体分子转动和振动减少, 能量损失少, 苂光增强 ${ }^{[13]}$. 而 $\mathrm{Cl}^{-}, \mathrm{Br}^{-}, \mathrm{I}^{-}, \mathrm{HSO}_{4}{ }^{-}$的加入对主体分子的苂光光谱无 明显影响. 同时, 受体分子 $\mathbf{4 a}$ 对 $\mathrm{F}^{-}$和 $\mathrm{AcO}^{-}$的检测限以 及量子产率进行测试, 检测限分别为 5.45 与 $7.00 \mu \mathrm{g} / \mathrm{L}$; 加入 5 equiv. 阴离子时量子产率最大增加为未加入阴离 子的 3 倍(表 $\mathrm{S} 1$ ). 表明受体分子对 $\mathrm{F}^{-}$或 $\mathrm{AcO}^{-}$有高选择 性以及低检测限.
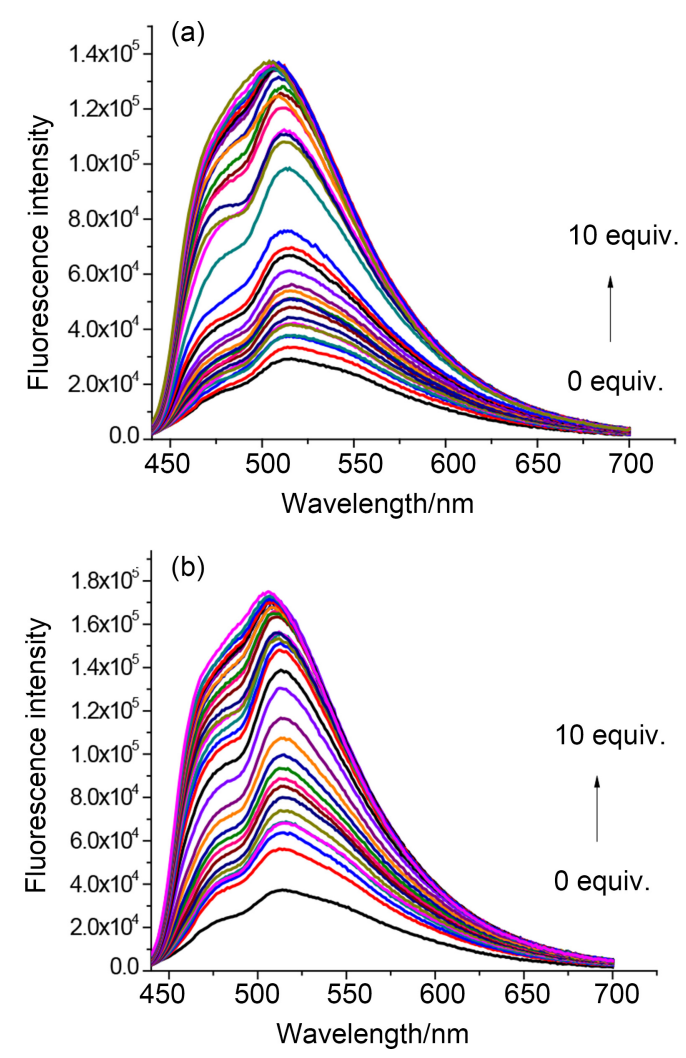

图 4 受体分子 $4 \mathbf{a}\left(c=2.5 \times 10^{-5} \mathrm{~mol} \cdot \mathrm{L}^{-1}\right)$ 在 $\mathrm{DMSO}$ 溶液的苂 光滴定曲线图

Figure 4 fluorescence spectroscopy titration of receptor $4 \mathbf{a}(c=$ $2.5 \times 10^{-5} \mathrm{~mol} \cdot \mathrm{L}^{-1}$ ) in DMSO solution

(a) $\mathrm{F}^{-}$; (b) $\mathrm{AcO}^{-}$

通过苂光光谱测定受体分子 $\mathbf{4 a}$ 对 $\mathrm{F}^{-}$和 $\mathrm{AcO}^{-}$响应时 间, 如图 5. 实验结果表明, 在 $30 \mathrm{~min}$ 内受体分子 $4 \mathrm{a}$ 的 苂光强度变化很小，表明受体分子 $\mathbf{4 a}$ 的苂光强度不受 时间的影响; 而加入 $\mathrm{F}^{-}$或 $\mathrm{AcO}^{-}$时, 体系的荧光强度逐 渐增强, 并逐渐趋于平稳, 测定受体分子 $\mathbf{4 a}$ 对 $\mathrm{F}^{-}$和 $\mathrm{AcO}^{-}$响应时间分别为 5 和 $10 \mathrm{~min}$.

\section{3 核磁滴定研究}

为了进一步研究受体分子 $\mathbf{4 a} \sim \mathbf{4 e}$ 对不同客体阴离 

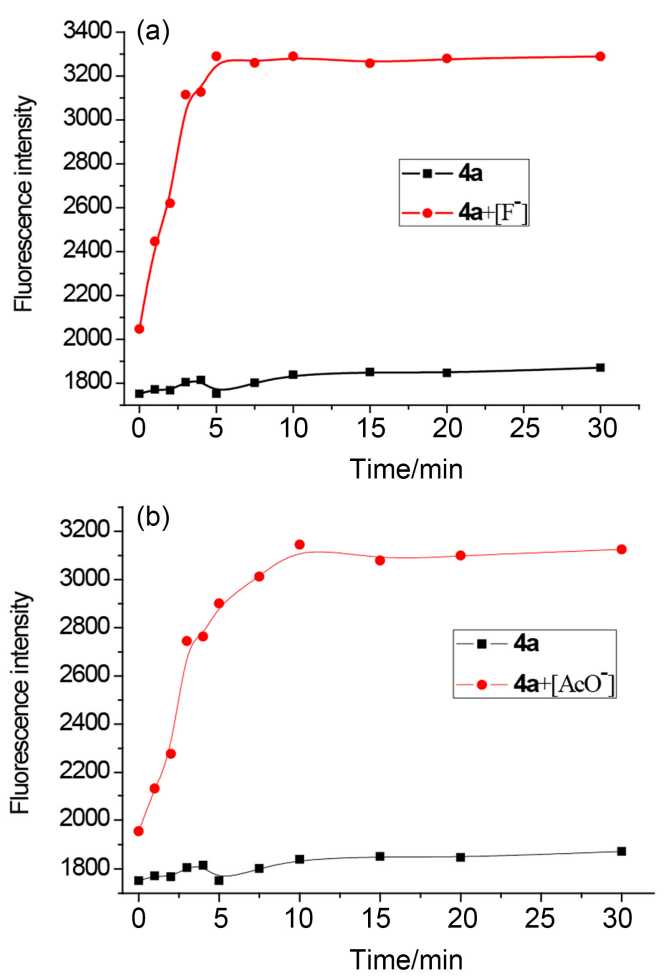

图 5 受体分子 $4 \mathrm{a}\left(c=2.5 \times 10^{-5} \mathrm{~mol} \cdot \mathrm{L}^{-1}\right)$ 在 DMSO 溶液的时 间响应

Figure 5 Effect reaction time of $\mathbf{4 a}\left(c=2.5 \times 10^{-5} \mathrm{~mol} \cdot \mathrm{L}^{-1}\right)$ in DMSO solution

$$
\text { (a) } \mathrm{F}^{-} \text {; (b) } \mathrm{AcO}^{-}
$$

子的亲和力. 通过核磁滴定实验考察了这些受体在 DMSO- $d_{6}$ 溶液中对 $\mathrm{F}^{-}, \mathrm{AcO}^{-}, \mathrm{Cl}^{-}, \mathrm{Br}^{-}, \mathrm{I}^{-}, \mathrm{HSO}_{4}{ }^{-}$后发生 的氢谱信号的变化, 如图 6. 实验表明, 只有滴加入 $\mathrm{F}^{-}$
或 $\mathrm{AcO}^{-}$阴离子时, 硫脲上 $\mathrm{N}$ 上的 $\mathrm{H}_{\mathrm{a}}, \mathrm{H}_{\mathrm{b}}, \mathrm{H}_{\mathrm{c}}$ 三个氢的化 学峰消失, $\mathrm{H}_{\mathrm{d}}$ 的化学位移向高场移动. 滴加 $\mathrm{Cl}^{-}, \mathrm{Br}^{-}, \mathrm{I}^{-}$ 和 $\mathrm{HSO}_{4}{ }^{-}$阴离子时, 化学位移的变化程度较小. 以受体 分子 $4 \mathrm{a}$ 和 $\mathrm{F}^{-}$的核磁滴定为例. 当滴加少量的 $\mathrm{F}^{-}$时, $\mathrm{F}^{-}$ 与受体通过氢键结合，受体分子 $\mathbf{4 a}$ 上 $\mathrm{H}_{\mathrm{a}}, \mathrm{H}_{\mathrm{b}}, \mathrm{H}_{\mathrm{c}}$ 三个氢 的化学位移峰强度减弱. 随着 $\mathrm{F}^{-}$的量增加, $\mathrm{F}^{-}$使得受体 分子 $4 \mathbf{a}$ 上 $\mathrm{H}_{\mathrm{a}}, \mathrm{H}_{\mathrm{b}}, \mathrm{H}_{\mathrm{c}}$ 去质子化，从而使得核磁 $\mathrm{H}_{\mathrm{a}}, \mathrm{H}_{\mathrm{b}}, \mathrm{H}$ 三个峰消失, 继续滴加 $\mathrm{F}^{-}$至 6 equiv. 时, 在 $\delta 16.3$ 处出现 三重峰，这说明受体分子去质子化产生了 $\mathrm{HF}_{2}{ }^{-}$. 去质子 化过程使得受体分子的电荷密度增高, 屏蔽效益增强, 因此 $\mathrm{H}_{\mathrm{d}}$ 化学位移向高场偏移.

\section{4 受体分子结合常数测定}

利用紫外吸收光谱, 采取连续法测定受体与阴离子 的配位常数 ${ }^{[14]}$, 绘制其 Job's 曲线, 确定受体分子 $\mathbf{4 a} \sim$ $\mathbf{4 e}$ 与 $\mathrm{F}^{-}$的结合比. 以受体分子 $\mathbf{4 a}$ 为例, 如图 7. 紫外可 见最大吸收峰落在横轴的 0.6 处, 表明受体分子 $\mathbf{4 a}$ 与 $\mathrm{F}^{-}$ 形成 $1: 2$ 的配合物, 并结合紫外滴定光谱的数据, 采用 最小二乘法曲线拟合, 求得受体 $\mathbf{4 a} \sim \mathbf{4 e}$ 与 $\mathrm{F}^{-}$的配位常 数如表 1 .

\section{5 识别机理探讨}

通过以上实验, 探究受体分子与 $\mathrm{F}^{-}$的作用机理 ${ }^{[15]}$, 受体分子 4a 本身分子内空间位阻较大，整个分子并不 是刚性的平面，而是存在一定角度，具有一定柔韧性， 这将更有利于 $\mathrm{F}^{-}$与受体结合. 核磁滴定实验表明，在 $\mathrm{F}^{-}$ 浓度较低时, $\mathrm{F}^{-}$与受体通过氢键作用相结合; 高浓度时, 由于氟离子的电负性较大，则发生质子化过程。如 Scheme 2 所示. 当向受体分子 $\mathbf{4 a}$ 滴加入低浓度 $\mathrm{F}^{-}$时,

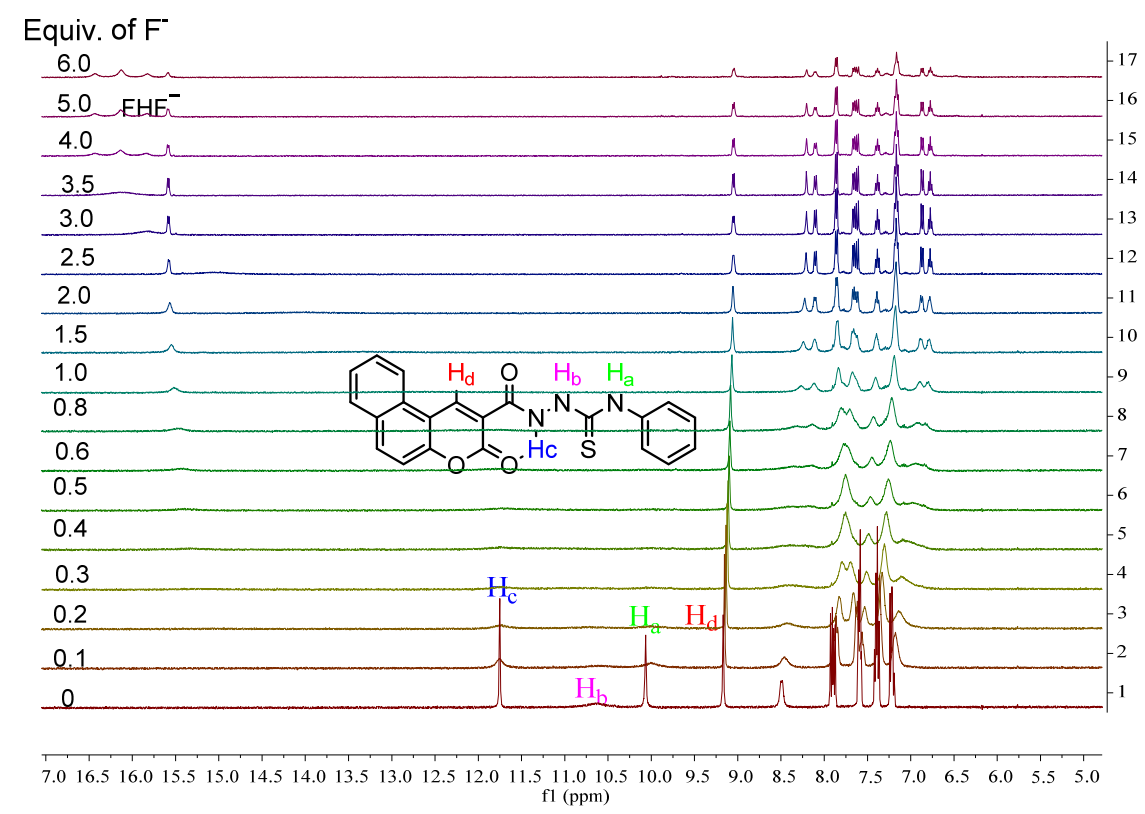

图 6 受体分子 $4 \mathrm{a}$ 在 DMSO- $d_{6}$ 溶液滴加 $\mathrm{F}^{-}$的核磁谱图

Figure $6{ }^{1} \mathrm{H}$ NMR spectra of receptor $4 \mathrm{a}$ in DMSO- $d_{6}$ with various concentrations of $\mathrm{F}^{-}$ 


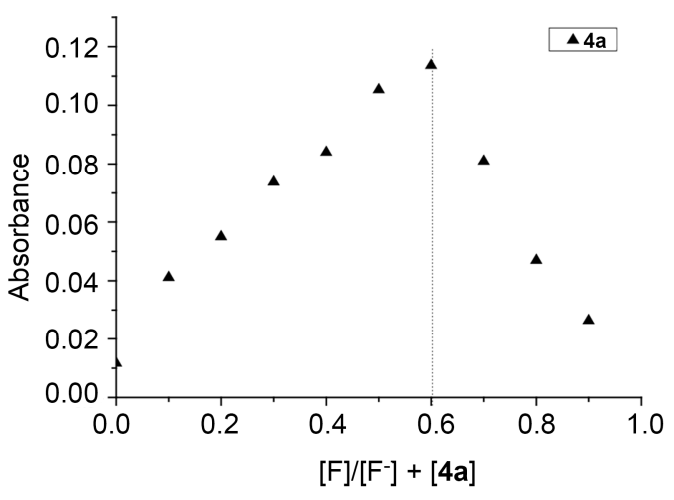

图 7 受体分子 $4 \mathbf{a}$ 与 $\mathrm{F}^{-}$作用的 Job's 曲线 $(\lambda=491 \mathrm{~nm})$ Figure 7 Job's plot between receptor $4 \mathbf{a}$ and fluoride anion in DMSO solution $(\lambda=491 \mathrm{~nm})$.

表 1 受体 $4 \mathrm{a} \sim 4 \mathrm{e}$ 对不同阴离子的结合常数 ${ }^{a}$

Table 1 Association constants $\left(K_{\mathrm{a}}\right)$ for $1: 2$ complexes of receptors $\mathbf{4 a} \sim \mathbf{4 e}$ with anions in DMSO at $298 \mathrm{~K}$

\begin{tabular}{lccccc}
\hline \multirow{2}{*}{ Anion $^{b}$} & \multicolumn{5}{c}{$K_{a} /\left(\mathrm{L}^{2} \cdot \mathrm{mol}^{-2}\right)$} \\
\cline { 2 - 6 } & $\mathbf{4 a}$ & $\mathbf{4 b}$ & $\mathbf{4 c}$ & $\mathbf{4 d}$ & $\mathbf{4 e}$ \\
\hline $\mathrm{F}^{-}$ & $7.94 \times 10^{9}$ & $1.44 \times 10^{9}$ & $8.51 \times 10^{7}$ & $3.16 \times 10^{8}$ & $8.91 \times 10^{8}$ \\
$\mathrm{AcO}^{-}$ & $5.01 \times 10^{9}$ & $5.01 \times 10^{8}$ & $6.61 \times 10^{7}$ & $1.07 \times 10^{8}$ & $6.02 \times 10^{8}$
\end{tabular}

${ }^{a}$ Errors are estimated to $b e<15 \%{ }^{b}$ The tetrabutylammonium salts are employed as the source of anions.<smiles>O=C(NNC(=S)Nc1ccccc1)c1cc2c(ccc3ccccc32)oc1=O</smiles>

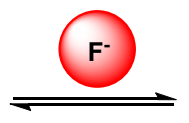<smiles>O=C(NNC(=S)Nc1ccccc1)c1cc2c(ccc3ccccc32)oc1=O</smiles>

图式 $24 a$ 与 $\mathrm{F}^{-}$作用机理图

Scheme 2 Proposed mechanism of receptor $4 \mathbf{a}$ interacted with $\mathrm{F}^{-}$

$\mathrm{F}^{-}$先与受体分子上活泼氢通过氢键作用相结合, 形成 $1: 2$ 的配合物, 一个 $\mathrm{F}^{-}$和硫嫝上两个 $\mathrm{N}-\mathrm{H}$ 氢键作用形 成六元环, 另外一分子 $\mathrm{F}^{-}$与酰胺上 $\mathrm{N}-\mathrm{H}$ 氢键作用. 随 着 $\mathrm{F}^{-}$量的增加, 受体分子 $\mathbf{4 a}$ 上三个活泼 $\mathrm{H}$ 发生去质子, 并有 $\mathrm{HF}_{2}{ }^{-}$生成, 使得分子内电荷密度增高, 分子的苂光 强度增强. 同样以受体分子 $\mathbf{4 a}$ 为例. 在受体分子 $\mathbf{4 a}$ 与 $\mathrm{F}^{-}$共存时 DMSO 溶液中, 溶液颜色呈现浅黄色. 向溶液
滴加少量质子性溶剂如甲醇等, 溶液颜色变浅逐步变回 受体分子 $4 \mathrm{a}$ 溶液的颜色, 这是由于 $\mathrm{F}^{-}$从受体中解离, 与游离的质子结合.

为了更好地解释受体分子与 $\mathrm{F}^{-}$的相互作用, 利用 高斯 09 中 B3LYP/6-31G(d,p)方法进行模拟, 优化结构 如图 8 和图 9, 重要键长数据如表 2. 受体分子 $\mathbf{4 a}$ 与阴 离子相互作用，受体分子硫脲的 N(7)和 N(9)上 $\mathrm{H}$ 先脱 去, 与 $\mathrm{F}^{-}$形成 $\mathrm{HF}_{2}{ }^{-}$, 使得分子内电荷转移, 从而导致硫

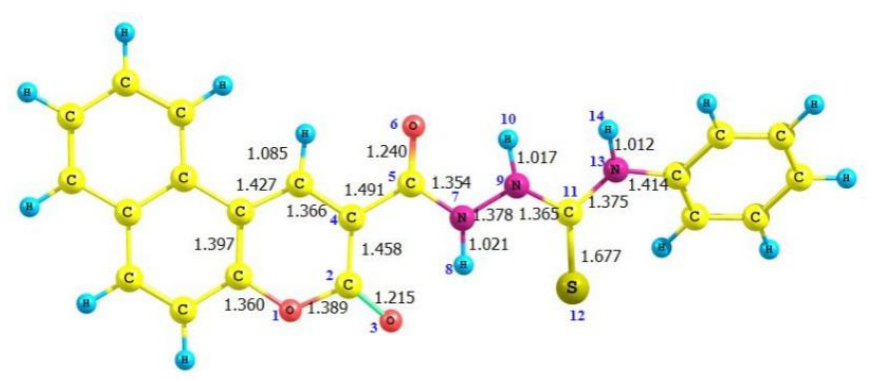

图 8 高斯 09 [B3LYP/6-31G (d, p)]方法计算出受体 4a 的优化 结构

Figure 8 DFT computed optimized structure of receptor $4 \mathbf{a}$ by applying B3LYP/6-31G (d, p) method

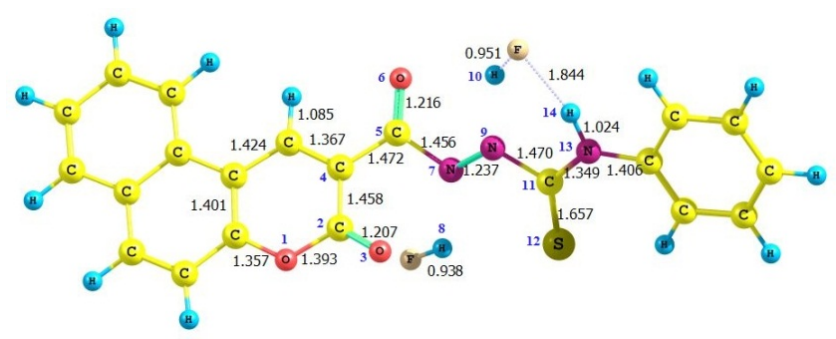

图 9 高斯 $09[\mathrm{~B} 3 \mathrm{LYP} / 6-31 \mathrm{G}(\mathrm{d}, \mathrm{p})]$ 方法计算出受体 $\mathbf{4 a}\left(\mathrm{F}^{-}\right)$的 优化结构

Figure 9 DFT computed optimized structure of receptor $4 \mathbf{a}\left(\mathrm{F}^{-}\right)$ by applying B3LYP/6-31G (d,p) method

表 2 采用 B3LYP/6-31G $(\mathrm{d}, \mathrm{p})$ 方法计算受体 $\mathbf{4 a}$ 以及 $\mathbf{4 a}\left(\mathrm{F}^{-}\right)$重 要键的键长 $(\AA)$

Table 2 DFT computed selected bond lengths $(\AA)$ for $4 \mathbf{a}$ and $\mathbf{4 a}\left(\mathrm{F}^{-}\right)$at B3LYP/6-31G(d, p) level

\begin{tabular}{lcl}
\hline Compd. & $\mathbf{4 a}$ & $\mathbf{4 a}\left(\mathrm{F}^{-}\right)$ \\
\hline $\mathrm{O}(1)-\mathrm{C}(2)$ & 1.389 & 1.393 \\
$\mathrm{C}(2)-\mathrm{O}(3)$ & 1.215 & 1.207 \\
$\mathrm{C}(2)-\mathrm{C}(4)$ & 1.458 & 1.458 \\
$\mathrm{C}(4)-\mathrm{C}(5)$ & 1.491 & 1.472 \\
$\mathrm{C}(5)-\mathrm{O}(6)$ & 1.240 & 1.216 \\
$\mathrm{C}(5)-\mathrm{N}(7)$ & 1.354 & 1.456 \\
$\mathrm{~N}(7)-\mathrm{H}(8)$ & 1.021 & None \\
$\mathrm{N}(7)-\mathrm{N}(9)$ & 1.378 & 1.237 \\
$\mathrm{~N}(9)-\mathrm{H}(10)$ & 1.017 & None \\
$\mathrm{N}(9)-\mathrm{C}(11)$ & 1.376 & 1.470 \\
$\mathrm{C}(11)-\mathrm{N}(13)$ & 1.375 & 1.349 \\
$\mathrm{~N}(13)-\mathrm{H}(14)$ & 1.012 & 1.024 \\
\hline
\end{tabular}


艮上 $\mathrm{C}-\mathrm{N}$ 键的键长增长, $\mathrm{N}-\mathrm{N}$ 键的键长缩短. 由 $\mathrm{C}(5)$ - $\mathrm{N}(7)$ 和 $\mathrm{N}(7)-\mathrm{N}(9)$ 的键长 1.354 和 $1.365 \AA$ 增长为 1.456 和 $1.456 \AA$, 分别增长了 0.102 和 $0.094 \AA$; 而 $\mathrm{N}(7)$ - $\mathrm{N}(9)$ 键长由 $1.378 \AA$ 缩短为 $1.237 \AA$, 缩短了 0.141 $\AA$.

\section{2 结论}

本文以 $\beta$-䒺酚为原料合成了 5 种具有不同电子效应 苯并香豆素硫艮类受体 $4 \mathbf{a} \sim 4 \mathbf{e}$, 并通过 ${ }^{1} \mathrm{H} N \mathrm{NMR},{ }^{13} \mathrm{C}$ NMR, IR 和元素分析等手段表征了化合物的结构. 通过 “裸眼识别” 实验, 当加入 $\mathrm{F}^{-}$或 $\mathrm{AcO}^{-}$时, 溶液颜色由无 色变为黄色. 紫外滴定实验表明, 分子 $4 \mathrm{a} \sim 4 \mathrm{e}$ 在 400 $\mathrm{nm}$ 处出现一个清晰的等吸收点, 形成稳定的配合物. 受体分子 $4 \mathrm{a} \sim 4 \mathrm{e}$ 与 $\mathrm{F}^{-}$或 $\mathrm{AcO}^{-}$作用时, 其荧光信号增 强, 这说明受体分子 $4 \mathrm{a} \sim 4 \mathrm{e}$ 对 $\mathrm{F}^{-}$和 $\mathrm{AcO}^{-}$形成稳定的化 合物. 通过 Job's 曲线可知受体分子 $\mathbf{4 a} \sim \mathbf{4 e}$ 与 $\mathrm{F}^{-}$形成 $1: 2$ 的配合物, 其配位常数 $K_{\mathrm{a}}$ 在 $10^{7} \sim 10^{9} \mathrm{~L}^{2} \cdot \mathrm{mol}^{-2}$ 之 间. 核磁滴定实验表明受体分子 $4 a \sim 4 e$ 含有三个 $\mathrm{N}-\mathrm{H}$ 作为阴离子识别位点, 与 $\mathrm{F}^{-}$或 $\mathrm{AcO}^{-}$配位, 响应好. 利 用高斯 $09[\mathrm{~B} 3 \mathrm{LYP} / 6-31 \mathrm{G}(\mathrm{d}, \mathrm{p})]$ 量化计算模拟受体分子 作用力, 进一步证实了受体分子与阴离子是通过氢键相 互作用. 受体分子 $\mathbf{4 a} \sim \mathbf{4 e}$ 可以选择性的识别 $\mathrm{F}^{-}$或 $\mathrm{AcO}^{-}$ 检测限分别为 5.45 和 $7.00 \mu \mathrm{g} / \mathrm{L}$, 响应时间分别为 5 和 10 min. 表明受体分子对 $\mathrm{F}^{-}$或 $\mathrm{AcO}^{-}$检测限低, 响应时 间短以及稳定性好等优点, 为发展 $\mathrm{F}^{-}$或 $\mathrm{AcO}^{-}$检测试纸 提供了理论依据.

\section{3 实验部分}

\section{1 仪器和试剂}

上海申光 WRS-1B 熔点仪, 温度未经校正; Bruker AVANCE III 400M 核磁共振仪 (400 MHz, TMS 为内标); Elementar Vario MICRO 元素分析仪; Thermo-FinnganLCQ 质谱仪; Spectrum 2000 傅立叶变换红外光谱仪 (KBr 压片); PerkinElmer Lambda 750 紫外光谱仪; HITACHI FLS-980 苂光光谱仪; HAMAMATSU C11347 量 子产率光谱仪. 3-甲酸-5,6-苯并吡喃酮按照文献[16, 17] 方法制备, 试剂均为分析纯. 紫外光谱实验试剂为光谱 纯.

\subsection{3-甲酰氯-5,6-苯并吡喃酮 3 的合成}

在带有干燥管, 回流冷凝管和吸收装置的干燥体系 中加入 3-甲酸-5,6-苯并吡喃酮 1 (9 mmol, 约 $2.2 \mathrm{~g}$ ), 加 入新制的二氯亚砜 $20 \mathrm{~mL}$, 搅拌, 加热至 $80{ }^{\circ} \mathrm{C}$, 反应 6 h. 常压蒸馏除去剩余的二氯亚砜, 即可得到黄色粉末 物质. 未经纯化直接进行下一步反应. 将除去 $\mathrm{SOCl}_{2}$ 残 留物中溶解于 $50 \mathrm{~mL}$ 吡啶. 缓慢滴加含有 $4 \mathrm{~mL}$ 水合肼
的吡啶溶液 $11 \mathrm{~mL}$, 在 $115{ }^{\circ} \mathrm{C}$ 下回流 $4 \mathrm{~h}$, 减压除去溶 剂, 用乙醇重结晶得到化合物 $\mathbf{3}$, 产量为 3.3 g. m.p. $259 \sim 260{ }^{\circ} \mathrm{C}$ (文献值 $260 \sim 261{ }^{\circ} \mathrm{C}$ ); ${ }^{1} \mathrm{H}$ NMR $(400 \mathrm{MHz}$, DMSO- $\left.d_{6}\right) \delta: 12.84(\mathrm{~s}, 1 \mathrm{H}, \mathrm{C}=\mathrm{CH}), 8.87(\mathrm{~s}, 1 \mathrm{H}, \mathrm{NH})$, $8.07(\mathrm{~d}, J=8.8 \mathrm{~Hz}, 1 \mathrm{H}, \mathrm{Ph}-\mathrm{H}), 7.84(\mathrm{~d}, J=8.0 \mathrm{~Hz}, 1 \mathrm{H}$, Ph-H), 7.76 (d, $J=8.8 \mathrm{~Hz}, 1 \mathrm{H}, \mathrm{Ph}-\mathrm{H}), 7.52$ (t, $J=8.0 \mathrm{~Hz}$, 1H, Ph-H), $7.39 \sim 7.32$ (m, J=8.4 Hz, 1H, Ph-H), 7.14 (d, $J=8.8 \mathrm{~Hz}, 1 \mathrm{H}, \mathrm{Ph}-\mathrm{H}), 7.02$ (s, $2 \mathrm{H}, \mathrm{NH}_{2}$ ).

\subsection{1-(5,6-苯并香豆素-3-甲酰基)-4-芳基氨基硫脲 $4 a \sim 4 e$ 的合成}

在 $250 \mathrm{~mL}$ 三口烧瓶中加入化合物 $\mathbf{3}(0.78 \mathrm{mmol}$, 约 $0.2 \mathrm{~g}$ )和苯甲酰基异硫氰酸酯 $(0.96 \mathrm{mmol}$, 约 $0.16 \mathrm{~g})$, 加 入 $50 \mathrm{~mL}$ 无水 1,4 -二氧六环使其溶解. 在 $105{ }^{\circ} \mathrm{C}$ 反应 5 h. 减压除去溶剂, 过柱, 二氯甲烷为洗脱剂, 得到黄色 粉末固体 4a, 产量为 $0.27 \mathrm{~g}$, 产率为 $88 \%$. m.p. 218 $220{ }^{\circ} \mathrm{C} .{ }^{1} \mathrm{H}$ NMR (400 MHz, DMSO- $\left.d_{6}\right) \delta: 11.78(\mathrm{~s}, 1 \mathrm{H}$, $\mathrm{N}-\mathrm{H}), 10.53(\mathrm{~s}, 1 \mathrm{H}, \mathrm{N}-\mathrm{H}), 10.09(\mathrm{~s}, 1 \mathrm{H}, \mathrm{N}-\mathrm{H}), 9.19$ (s, 1H, PhC-H), 8.50 (s, 1H, Ph-H), 7.90 (dd, $J=15.0,8.4 \mathrm{~Hz}$, $2 \mathrm{H}, \mathrm{Ph}-\mathrm{H}$ ), 7.59 (t, $J=8.0 \mathrm{~Hz}, 3 \mathrm{H}, \mathrm{Ph}-\mathrm{H}$ ), 7.39 (dt, $J=7.6$, $3.5 \mathrm{~Hz}, 3 \mathrm{H}, \mathrm{Ph}-\mathrm{H}), 7.22$ (dd, $J=14.4,8.1 \mathrm{~Hz}, 2 \mathrm{H}, \mathrm{Ph}-\mathrm{H}$ ); ${ }^{13} \mathrm{C}$ NMR (100 MHz, DMSO- $\left.d_{6}\right) \delta$ : 177.21, 167.91, $158.72,151.83,134.09,133.66,132.46,132.40,129.33$, $129.23,128.98,128.24,128.21,124.16,121.64,119.60$, 108.93; IR (KBr) v: $1624(\mathrm{C}=\mathrm{C}), 1604(\mathrm{C}=\mathrm{O}), 1265(\mathrm{C}$ $=\mathrm{S}) \mathrm{cm}^{-1}$; MS (ESI) $\mathrm{m} / z: 390.48[\mathrm{M}+\mathrm{H}]^{+}$. Anal. calcd for $\mathrm{C}_{21} \mathrm{H}_{15} \mathrm{~N}_{3} \mathrm{O}_{3} \mathrm{~S}$ : C 64.77, $\mathrm{H}$ 3.88, N 10.79; found $\mathrm{C}$ 65.04, H 3.63, N 10.70 .

受体分子 $\mathbf{4 b}$ 的制备方法类似于受体分子 $\mathbf{4 a}$, 黄色 粉末, 产量为 $0.20 \mathrm{~g}$, 产率为 $63 \%$. m.p. $180 \sim 182{ }^{\circ} \mathrm{C} ;{ }^{1} \mathrm{H}$ NMR (400 MHz, DMSO- $\left.d_{6}\right) \delta: 11.70(\mathrm{~s}, 1 \mathrm{H}, \mathrm{N}-\mathrm{H}), 10.63$ (s, 1H, N-H), $10.01(\mathrm{~s}, 1 \mathrm{H}, \mathrm{N}-\mathrm{H}), 9.18(\mathrm{~s}, 1 \mathrm{H}, \mathrm{PhC}-\mathrm{H})$, 8.47 (s, 1H, Ph-H), 7.89 (dd, $J=13.8,8.5$ Hz, 2H, Ph-H), 7.59 (t, $J=7.7 \mathrm{~Hz}, 1 \mathrm{H}, \mathrm{Ph}-\mathrm{H}), 7.49 \sim 7.31$ (m, 2H, Ph-H), 7.39 (dd, $J=14.4,7.0 \mathrm{~Hz}, 1 \mathrm{H}, \mathrm{Ph}-\mathrm{H}), 7.23$ (d, $J=8.9 \mathrm{~Hz}$, 1H, Ph-H), 7.19 (d, $J=8.0 \mathrm{~Hz}, 2 \mathrm{H}, \mathrm{Ph}-\mathrm{H}$ ), 2.30 (d, $J=12.5$ $\left.\mathrm{Hz}, 3 \mathrm{H}, \mathrm{CH}_{3}\right)$; IR $(\mathrm{KBr}) v: 1624(\mathrm{C}=\mathrm{C}), 1598(\mathrm{C}=\mathrm{O})$, $1261(\mathrm{C}=\mathrm{S}) \mathrm{cm}^{-1}$; MS (ESI) $\mathrm{m} / z: 404.11[\mathrm{M}+\mathrm{H}]^{+}$. Anal. calcd for $\mathrm{C}_{22} \mathrm{H}_{17} \mathrm{~N}_{3} \mathrm{O}_{3} \mathrm{~S}$ : C 65.49, H 4.25, N 10.42; found C 65.18 H 3.39, N 10.51 .

受体分子 $4 \mathrm{c}$ 的制备方法类似于受体分子 $4 \mathrm{a}$, 黄色 粉末, 产量为 $0.27 \mathrm{~g}$, 产率为 $83 \%$. m.p $182 \sim 184{ }^{\circ} \mathrm{C} ;{ }^{1} \mathrm{H}$ NMR (400 MHz, DMSO- $\left.d_{6}\right) \delta: 12.07(\mathrm{~s}, 1 \mathrm{H}, \mathrm{N}-\mathrm{H}), 10.65$ (s, 1H, N-H), $10.36(\mathrm{~s}, 1 \mathrm{H}, \mathrm{N}-\mathrm{H}), 9.18(\mathrm{~s}, 1 \mathrm{H}, \mathrm{PhC}-\mathrm{H})$, 8.54 (s, 1H, Ph-H), 7.92 (dd, J=17.7, 8.0 Hz, 2H, Ph-H), $7.87(\mathrm{~d}, J=7.9 \mathrm{~Hz}, 1 \mathrm{H}, \mathrm{Ph}-\mathrm{H}), 7.83(\mathrm{~d}, J=8.4 \mathrm{~Hz}, 2 \mathrm{H}$, 
Ph-H), 7.79 (s, 1H, Ph-H), 7.58 (t, $J=7.7$ Hz, 1H, Ph-H), 7.40 (t, $J=7.3 \mathrm{~Hz}, 1 \mathrm{H}, \mathrm{Ph}-\mathrm{H}), 7.24$ (d, $J=8.9 \mathrm{~Hz}, 1 \mathrm{H}$, Ph-H); IR (KBr) v: $1621(\mathrm{C}=\mathrm{C}), 1608(\mathrm{C}=\mathrm{O}), 1270(\mathrm{C}=$ S) $\mathrm{cm}^{-1}$; MS (ESI) $m / z$ : $413.47[\mathrm{M}-\mathrm{H}]^{+}$. Anal. calcd for $\mathrm{C}_{22} \mathrm{H}_{14} \mathrm{~N}_{4} \mathrm{O}_{3} \mathrm{~S}$ : C 63.76, H 3.41, N 13.52; found C 64.09, H 3.41, N 14.02 .

受体分子 $\mathbf{4 d}$ 的制备方法类似于受体分子 $\mathbf{4 a}$, 黄色 粉末, 产量为 $0.22 \mathrm{~g}$, 产率为 $67 \%$. m.p. 207 $209{ }^{\circ} \mathrm{C} ;{ }^{1} \mathrm{H}$ NMR (400 MHz, DMSO- $\left.d_{6}\right) \delta: 11.66(\mathrm{~s}, 1 \mathrm{H}, \mathrm{N}-\mathrm{H}), 10.62$ (s, 1H, N-H), 9.97 (s, 1H, N-H), $9.18(\mathrm{~s}, 1 \mathrm{H}, \mathrm{PhC}-\mathrm{H})$, $8.47(\mathrm{~d}, J=6.8 \mathrm{~Hz}, 1 \mathrm{H}), 7.97 \sim 7.81(\mathrm{~m}, 2 \mathrm{H}, \mathrm{Ph}-\mathrm{H}), 7.64 \sim$ 7.55 (m, 1H, Ph-H), 7.46 7.37 (m, 2H, Ph-H), 7.36 7.30 (m, 1H, Ph-H), 7.23 (d, J=8.9 Hz, 1H, Ph-H), 7.02 6.94 (m, 2H, Ph-H), 6.92 6.87 (m, 1H, Ph-H), $3.76(\mathrm{~d}, J=$ $12.0 \mathrm{~Hz}, 3 \mathrm{H})$; IR $(\mathrm{KBr}) v: 1623(\mathrm{C}=\mathrm{C}), 1598(\mathrm{C}=\mathrm{O})$, $1264(\mathrm{C}=\mathrm{S}) \mathrm{cm}^{-1}$; MS (ESI) $\mathrm{m} / z: 418.38[\mathrm{M}-\mathrm{H}]^{+}$. Anal. calcd for $\mathrm{C}_{22} \mathrm{H}_{17} \mathrm{~N}_{3} \mathrm{O}_{4} \mathrm{~S}$ : C 63.00, H 4.09, N 10.02; found C 62.82, H 3.62, N 10.41 .

受体分子 $4 \mathrm{e}$ 的制备方法类似于受体分子 $4 \mathrm{a}$, 黄色 粉末, 产量为 $0.28 \mathrm{~g}$, 产率为 $82 \%$. m.p. $194 \sim 196{ }^{\circ} \mathrm{C} ;{ }^{1} \mathrm{H}$ NMR (400 MHz, DMSO- $\left.d_{6}\right) \delta: 12.15(\mathrm{~s}, 1 \mathrm{H}, \mathrm{N}-\mathrm{H})$, $11.00 \sim 10.07(\mathrm{~m}, 2 \mathrm{H}, \mathrm{N}-\mathrm{H}), 9.19$ (s, 1H, PhC-H), 8.55 (s, 1H, Ph-H), 8.23 (t, $J=12.6 \mathrm{~Hz}, 2 \mathrm{H}, \mathrm{Ph}-\mathrm{H}), 8.17$ (s, $1 \mathrm{H}$, Ph-H), 8.03 (d, $J=8.5 \mathrm{~Hz}, 2 \mathrm{H}, \mathrm{Ph}-\mathrm{H}), 7.92$ (d, $J=8.9 \mathrm{~Hz}$, 1H, Ph-H), 7.87 (d, $J=7.8 \mathrm{~Hz}, 1 \mathrm{H}, \mathrm{Ph}-\mathrm{H}), 7.59$ (t, $J=7.5$ $\mathrm{Hz}, 1 \mathrm{H}, \mathrm{Ph}-\mathrm{H}), 7.40$ (t, $J=7.3 \mathrm{~Hz}, 1 \mathrm{H}, \mathrm{Ph}-\mathrm{H}), 7.22$ (dd, $J=14.8,9.0 \mathrm{~Hz}, 1 \mathrm{H}, \mathrm{Ph}-\mathrm{H})$; IR $(\mathrm{KBr}) v: 1622(\mathrm{C}=\mathrm{C})$, $1596(\mathrm{C}=\mathrm{O}), 1260(\mathrm{C}=\mathrm{S}) \mathrm{cm}^{-1}$; MS (ESI) $\mathrm{m} / z: 432.99$ $[\mathrm{M}-\mathrm{H}]^{+}$. Anal. calcd for $\mathrm{C}_{21} \mathrm{H}_{14} \mathrm{~N}_{4} \mathrm{O}_{5} \mathrm{~S}$ : C 58.06, H 3.25, N 12.90; found C 58.41, H 3.37, N 13.05.

\section{4 紫外滴定测试}

PerkinElmer Lambda 750 紫外光谱仪, 在光谱纯的 DMSO 溶剂中测试, 将受体分子 $\mathbf{4 a} \sim \mathbf{4} \mathbf{e}$ 配制成浓度为 $2.5 \times 10^{-5} \mathrm{~mol} \cdot \mathrm{L}^{-1}$ 溶液. 受体分子的浓度保持不变, 利 用滴加法逐次加入四丁基铵盐的阴离子, 观察其受体分 子与阴离子结合后紫外可见光谱变化.

\section{5 荧光滴定测试}

HITACHI FLS-980 苂光光谱仪, 在光谱纯的 DMSO 溶剂中测试, 将受体分子 $\mathbf{4 a} \sim \mathbf{4 e}$ 配制成浓度为 $2.5 \times$ $10^{-5} \mathrm{~mol} \cdot \mathrm{L}^{-1}$ 溶液. 受体分子的浓度保持不变, 以 410 $\mathrm{nm}$ 为激发波长, 扫描发射谱, 利用滴加法逐次加入四
丁基铵盐的阴离子，观察其受体分子与阴离子结合后荧 光光谱变化.

\section{$3.6{ }^{1} \mathrm{H}$ NMR 滴定测试}

Bruker AVANCE III 400M 核磁共振仪, DMSO- $d_{6}$ 溶 液中测试，在受体分子 $\mathbf{4 a} \sim \mathbf{4 e}\left(2.5 \times 10^{-5} \mathrm{~mol} \cdot \mathrm{L}^{-1}\right)$ 中利 用累积滴加法逐次加入四丁基铵盐的阴离子, 观察其受 体分子与阴离子结合后活泼氢化学位移的变化.

辅助材料(Supporting Information) 受体分子 $4 \mathrm{a} \sim 4 \mathrm{e}$ 的 NMR 谱图, 以及受体与不同阴离子紫外、苂光、核 磁滴定堆积图. 这些材料可以免费从本刊网站 (http://sioc-journal.cn/)上下载.

\section{References}

[1] Jiang, X. Z.; Feng, M. Y.; Zhang, D. W.; Wang, B. S.; Dong, Z. Y.; Gao, G. H. Chin. J. Chem. 2013, 31, 673.

[2] Yang, Z. P.; Zhang, K.; Gong, F. B.; Li, S. Y.; Chen, J.; Ma, J. S.; Sobenina, L. N.; Mikhaleva, A. I.; Trofimov, B. A.; Yang, G. Q. J. Photochem. Photobiol., A 2011, 217, 29.

[3] Liu, R. Y.; Yong, X.; Yang, X. D.; Yan, Y. S.; Lu, X. W.; Qu, J. Q. Chin. J. Org. Chem. 2014, 34, 561 (in Chinese).

(刘瑞源, 勇雪, 杨小东, 严轶琛, 路新卫, 翟金清, 有机化学, 2014, 34, 561.)

[4] Chen, H. M.; Yue, F.; Lin, H. K. Chem. J. Chin. Univ. 2012, 33(6), 1239 (in Chinese).

(陈华梅, 岳凡, 林华宽, 高等学校化学学报, 2012, 33(6), 1239.)

[5] Zhang, Y. M.; Wang, A. X.; Cao, C.;Leng, Y. L.; Wei, T. B. Chin. J. Chem. 2009, 27, 1617.

[6] Çakı1, E. Ind. Eng. Chem. Res. 2000, 39(10), 3582.

[7] Brunet, E.; García-Losada, P.; Rodríguez-Ubis, J. C.; Juanes, O. Can. J. Chem. 2002, 80(2), 169.

[8] Peng, M. S.; Cai, J. Dyes Pigm. 2008, 79(3), 270.

[9] Maity, D.; Govindaraju, T. Inorg. Chem. 2010, 49(16), 7229.

[10] Zhang, Q.; Zhu, Z.; Zheng, Y.; Zheng, Y. L.; Cheng, J. G.; Zhang, N.; Long, Y. T.; Zheng, J.; Qian, X. H.; Yang, Y. J. J. Am. Chem. Soc. 2012, 134(45), 18479.

[11] Zhou, Y.; Zhang, J. F.; Yoon, J. Chem. Rev. 2014, 114(10), 5511.

[12] Yadav, U. N.; Pant, P.; Sharma, D.; Sahoo, S. K.; Shankarling, G. S. Sens. Actuators, B 2014, 197, 73.

[13] Qian, L. J.; Tong, B.; Shen, J. B.; Shi, J. B.; Zhi, J. G.; Dong, Y. Q.; Yang, F.; Dong, Y. P.; Lam, J. W. Y.; Liu, Y.; Tang, B. Z. Phys. Chem. B 2009, 113, 9089

[14] Pocker, Y.; Ciula, J. C. J. Am. Chem. Soc. 1989, 111(13), 4728.

[15] Li, S. W.; Cao, X. F.; Chen, C. S.; Ke, S. Y. Spectrochim. Acta, Part A 2012, 96, 18.

[16] Zhang, H.; Yu, T. Z.; Zhao, Y. L.; Fan, D. W.; Qian, L.; Yang, C. H.; Zhang, K. Spectrochim. Acta, Part A 2007, 68(3), 725.

[17] Maggi, R.; Bigi, F.; Carloni, S.; Mazzacani, A.; Sartori, G. Green Chem. 2001, 3(4), 173. 